\title{
A Simplified Outpatient Health Monitoring System in Resource Constrained IoT Infrastructure
}

\author{
Stanley Leonard Tito \\ Department of Information and Communication Technology \\ Mbeya University of Science and Technology (MUST), Mbeya, Tanzania
}

\begin{abstract}
Health monitoring is an essential task in managing both the daily routine progress of a patient/outpatient during and after the medication period. It plays not only a significant role in the overall well-being of the patient/outpatient but also as the measure of quality and effective healthcare services deliverance. However, achieving this is very challenging in areas with limited infrastructure to support a myriad of current social services, including advanced healthcare services. For instance, instead of relying only on the outpatient's physical visitations to the healthcare center as a feedback mechanism in case of any complications during medications, continuously remote monitoring of the outpatients' health can improve the deliverance of healthcare services. This paper presents a simplified system model for remote monitoring of the outpatients well-being that has been designed to work in the limited Internet of Things (IoT) infrastructure to address the challenge above. The design is cheap and easily deployable for health remote monitoring purposes as the outpatient's well-being can be monitored in real-time so that to facilitate detection of the subtle changes and avoid drug intoxication. The system advises and alerts in real-time the doctors/medical assistants about the changing of vital parameters in order to take preventive measures.
\end{abstract}

Keywords: Health Care System, Remote Monitoring System, Internet of Things, Short Message Service, Sensors, Body Area Network.

\subsection{INTRODUCTION}

One of the social and economic challenges that most of the countries face globally is how to improve the healthcare system as the population is growing. Of the past decades, an increased number of elderly and people who suffer various chronic noncommunicable diseases have brought more burden and pressure on improving the health care system [1-2]. The health care system comprises of either interaction of humans, the technology application, or, most commonly, altogether [3]. Typically, this is through services provisioning from health care providers whose collective goals are: prevention of poor health, raising standards in the quality, safety, and precision of care and medicine, making healthcare deliverance more proficient and cost-effective. In this context, the provisioning of enhanced and reliable health care services relies on the appropriate allocation of the available resources. One of the critical and significant resources that need efficient management strategy in healthcare services deliverance is physician supply in terms of the ratio of physicians to patients (i.e., how many patients at one time that one physician provides) [4]. Physician supply is a phrase that is used to specify the number of physicians with qualified training who work within the healthcare system or the labor market at large [5].

More specifically, the doctor to patient ratio (DPR), which refers to a number of doctors assigned to the patients, is one of the vital element in achieving an efficient and reliable health care system. As per the World Health Organization (WHO), the recommended DPR is 1/1000 [6]. However, the ratio is unachievable in most healthcare systems within several countries due to the increasing population growth rate, which outweighs the number of doctors' enrolment within the healthcare system. For instance, in Tanzania, the DPR ratio is $1 / 10,000$, which reflects the same case among developing countries with constrained healthcare system infrastructure [7, 9]. This situation does not comply with the DPR as per WHO recommendation, hence the necessity of taking immediate efficient measures to address the situation. 
Proactively, offloading some tasks to patients in the monitoring process can be of great advantage. In this regard, the outpatients (of which this system is specially designed for) are in charge of taking care of their health systematically through the use of new information and communication technologies (ICT) [10-11]. The healthcare industry is rapidly transforming into coordinated and user-centric systems. This digital transformation implies advanced integration and interoperable healthcare services. Centralized access to health records and patients related information focus to improve patient's healthcare monitoring and provisioning[11, 13].

Traditionally, in the monitoring of the outpatients' well-being relies on the physical feedback mechanism from the outpatients. The outpatients must frequently report to the healthcare center, their well-being progress during and after the medications period in case of any health complication [14]. This approach is an inefficient monitoring mechanism, as it consumes a lot of time and energy for both the outpatients and the healthcare givers. Additionally, it does not fetch and report essential data necessary for making appropriate decisions on time, which can lead to severe complications of the outpatients' health. Hence, using automation can improve the inefficient feedback mechanism through the application of technology, such as the usage of ICT, particularly the Internet of things (IoT) $[11,13,15]$. In this context, healthcare services deliverance can be improved through IoT application by remote monitoring of the outpatients' health in real-time and reacting appropriately.

IoT is an advancing Internet from the network of computers to the network of things or objects[16, 20]. The connected smart objects interact, collect, and share data in the background, which, in the end, creates useful information for an end-user or influences the end user's actions [21]. As shown in figure 1, it promises a wide range of applications in our daily routine activities. It is capable of connecting remote things, machines, or mobile devices by using wireless infrastructure[22, 25]. IoT infrastructure supports proficiently monitoring and controlling different activities within the surroundings[26, 31]. In this regard, through sensors and modern interconnecting technologies, necessary health data from the outpatients can be dynamically collected and analyzed to foster preventive care, diagnostics, and even measure treatment results [32]. Currently, where IoT is deployed and embraced plays a significant role in improving health care services. However, the adoption of technology, especially in developing countries, is a challenge due to poor infrastructure to support the technology. This paper presents a simplified and cost-effective outpatients' health monitoring system in an IoT constrained infrastructure by leveraging the GSM infrastructure and ubiquitous gadgets such as smart watches and fitness wristbands for improving healthcare services deliverance. The focus to surveys several RHMS and their architecture, suggest the flexible, simplified RHMS for constrained IoT infrastructure through leveraging the existing resources and infrastructure and also gives insights for future directions and improvement in RHMS deployment in constrained environments.

The structure for the rest of the paper is as follows. Section II discusses a general background of RHSM and reviews the related works regarding RHMS application in the IoT ecosystem. In Section III, presents the simplified outpatient RHMS design. Section IV gives a comparative evaluation of the ideal RHMS over the proposed simplified one. In Section V, concludes the paper and highlights recommendations for future work.

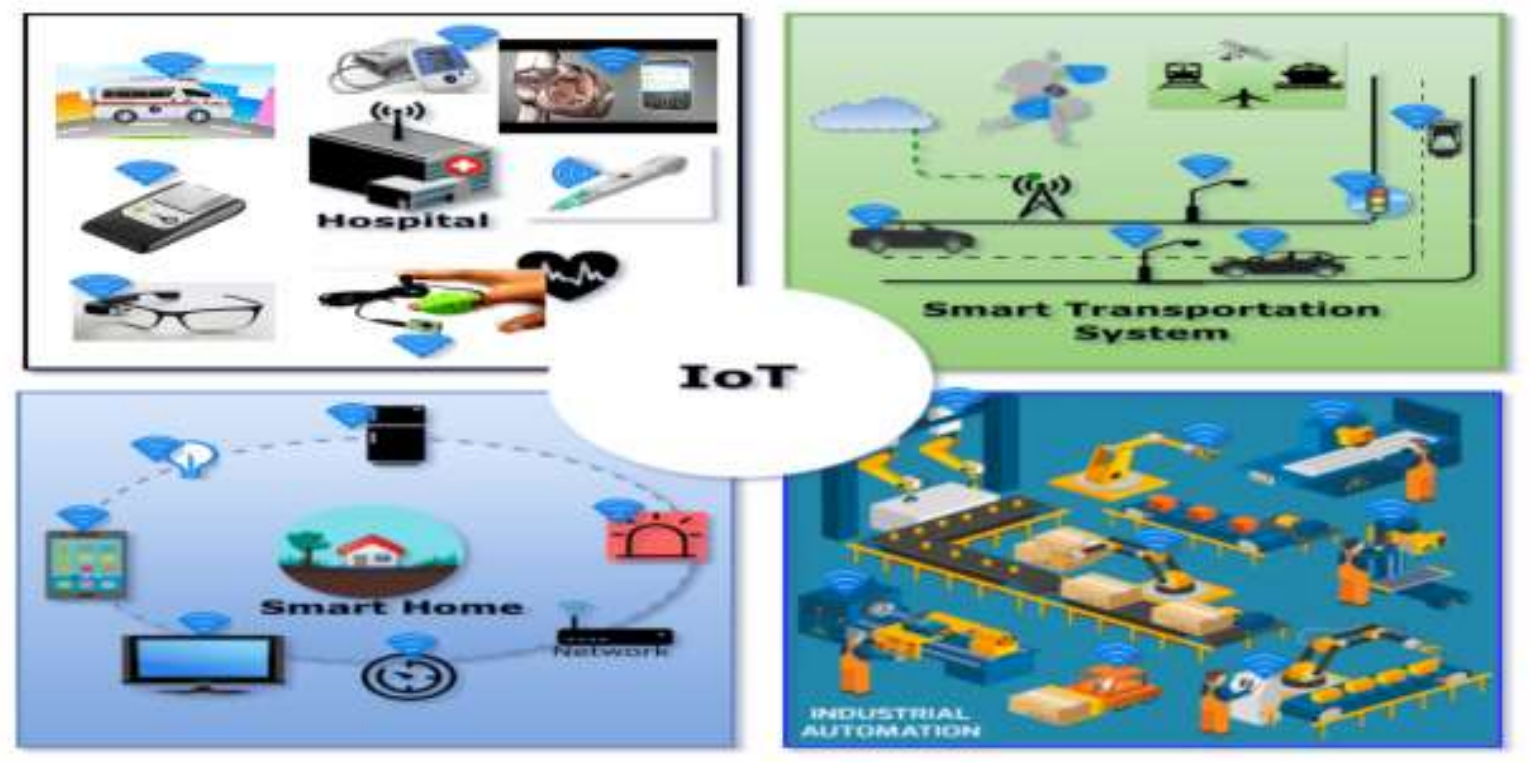

Figure 1. Example of IoT use cases [5] 


\subsection{Overview of IoT Application in Remote Health Monitoring}

The IoT forms a connected set of things at anyplace and anytime thus can provide access to anyone, any network and any service $[10,33]$. Health care represents one of the most attractive use case areas for the IoT [10,34,35]. Remote health monitoring for elderly care and chronic diseases is one of the significant roles that IoT plays in medical applications. Another critical application is healthcare providers' compliance regarding the case of medication and treatment at home. Consequently, several medical devices, sensors, imaging and diagnostic devices are smart devices or objects which form an essential part of the IoT application in healthcare.

Smart internet-connected devices find increasingly more applications within and outside the walls of the medical institution all over the world. Currently, worldwide, there are installations comprise of more than 100 million healthcare IoT devices, and this will keep on increasing [36, 37]. This interconnection of IoT medical devices helps the machine to machine (M2M) interaction and real-time data streaming between devices and healthcare centers $[38,40]$. IoT-based healthcare services promise to decrease costs, improve the quality of life, and the experience of the user. From the viewpoint of healthcare providers, the IoT has the potential to lessen device downtime by remote provision.

Besides, through IoT application, we can precisely know optimum times for provisioning various devices for their continuous and smooth operation. Moreover, the use of IoT provides effective scheduling of inadequate resources by guaranteeing better usage of available services for more patients through remote medical assistance, tracking staff, patients and inventory, Operations argumentations, data insights, drugs memorial et cetera. Figure 2 illustrates recent healthcare trends [11].The majority of health software applications (Apps) available in smart phones focus primarily on weight loss and overall fitness. However, in the domain of healthcare, there are smart phone apps designed to track symptoms, emotions, and sleep patterns, while others will manage prescriptions and health records or keep user's mind active with plenty of puzzles and games.

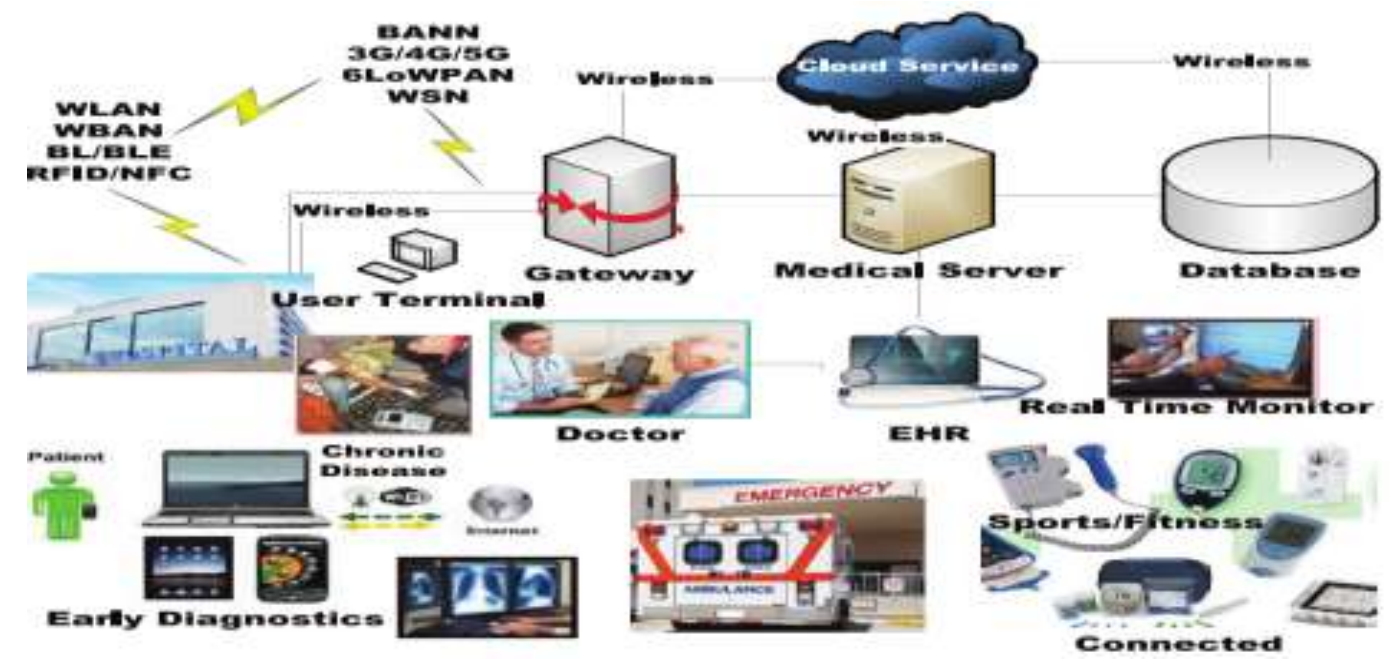

Figure 2. Current healthcare trends in IoT application[11]

Despite all these merits, the IoT based healthcare industry also faces several challenges like innovation cost, trivial data outpouring, data security and privacy, knowledge and skills on how to use the IoT based technologies. Analysis development and implementation of IoT based health care system require a profound investment and clear understanding of its purpose on the part of the hospital management [13].

Data outpouring inexperience and knowledge hold back the use of IoT connected devices in the healthcare industry, especially in rural areas. The right way to process the large volume of data from IoT devices is vital before storing it for further processing and analytics.

Security and privacy of patients and managerial data is still a challenge to most of the healthcare industries. With the use of IoT technology, security issues can further disappoint if cyber-attacks will influence patients' health directly. This case can happen since the majority of the connected medical devices do not comply with the data protection standards. Data usage can be for good or evil intent since the patient's data will be streamed, viewed, stored and analyzed all the time. Knowledge and skills on how to work and use this emerging IoT technology also seem to be a challenge not only to end-users but also to medical professionals as well [11-13]. 
To whatever degree these challenges posing difficultness in achieving a perfect solution, it should not be the main reason for not adopting and harnessing IoT applications in Healthcare systems. Upon trading off, it is clear that benefits outweigh challenges in a reasonable margin. For instance, in worse case scenarios where remote health monitoring is vital for general life safety and wellbeing of people, adopting a simplified model can be a significant step as a transition strategy towards a complete IoT model in delivering healthcare services. This paper emphasis on this aspect by leveraging ubiquitous computing in enabling remote health monitoring to improve healthcare services deliverance in local remote areas with limited ICT infrastructure and healthcare services.

\subsection{RELATED WORK}

The remote healthcare monitoring system architecture must reflect the core functionality of the RHMS which is to continuously measure and analyze mobile patient's bio signals from a distance by employing mobile computing, wireless communications and network technologies [41]. In this context, the RHMS comprises of sensors which are ported over the patient body to collect patient's medical data such as temperature, Blood pressure, heart rate, oxygen level, ECG, body temperature, sweating, falling et cetera[12,13,15, 42]. Subsequently, sensors forward the collected data to the designated endpoint through a dedicated communication gateway or directly to the server for analysis and diagnostics. In an ideal RHMS, the infrastructure goes beyond the one as mentioned earlier. It is complicated as it must be flexible, secure, reliable and support the seamless flow of the information between the remote data center and servers with certain assured quality levels. Fig. 3 shows the generic architecture of the RHMS[42].

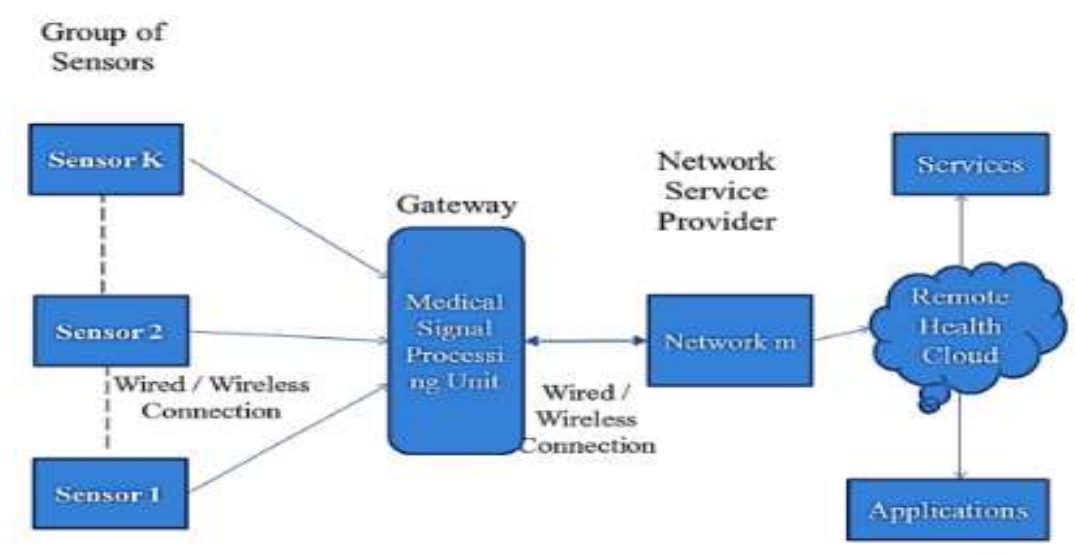

Figure 3. A generic architecture of the RHMS[42]

As proposed in $[43,44]$ the genetic architecture of the RHMS consists of a group of sensors or sensors end devices are used as a primary source for medical data collection from the patient body. Intra sensors network communication may or may not be present depending on the reusability, scalability and deployment scenario of the health care services. In order to have a system more open and reconfigurable, it is recommended to have sensors intra-network communication provisioned within remote health care network. Patients' medical data will be transmitted to the intermediate gateway via short-range technologies like Bluetooth. The gateway may perform data aggregation, filtering and forwarding to the next tier, i.e. back end server. The gateway can be dedicated hardware or a general handheld small device configured for the purpose like Zigbee coordinator or PDA/smart phone. Medical data from the gateway is forwarded to the remote end health care server or cloud services. Other stakeholders of the systems access and monitor the patient data from the cloud only. Auxiliary emergency services like alert/SMS to patient and caregivers may be augmented.

Depending on the operation and deployment requirements, the generic RHMS architecture can be further broken down into three standard groups. The groups are basing on the network access strategy used to facilitate end to end communication, which are: "No Network Access System", "Direct Network Access System" and "Network Access through Gateway" [42]

In the first group, i.e. "No Network Access System" as shown in figure 4 refers to standalone closed systems. The systems which store health-related information within the PDA/Smart phone or any smart gadgets. No-network access remote health monitoring systems. Such systems are reporting and generating alert for the concern patients or caregiver only. Entire data, analytic engines and other utilities reside within the patient owned device designed dedicatedly for the purpose. In[45, 47] authors have proposed RHMS basing on this architecture. These Healthcare systems use smart phone/PDA as a back end. These devices determine the result and provide health-related solutions. Sensors ported on the patient's body, or smart phone / PDA accumulate their medical data and transmit the data to the smart phone/PDA via either wired or wireless medium. 


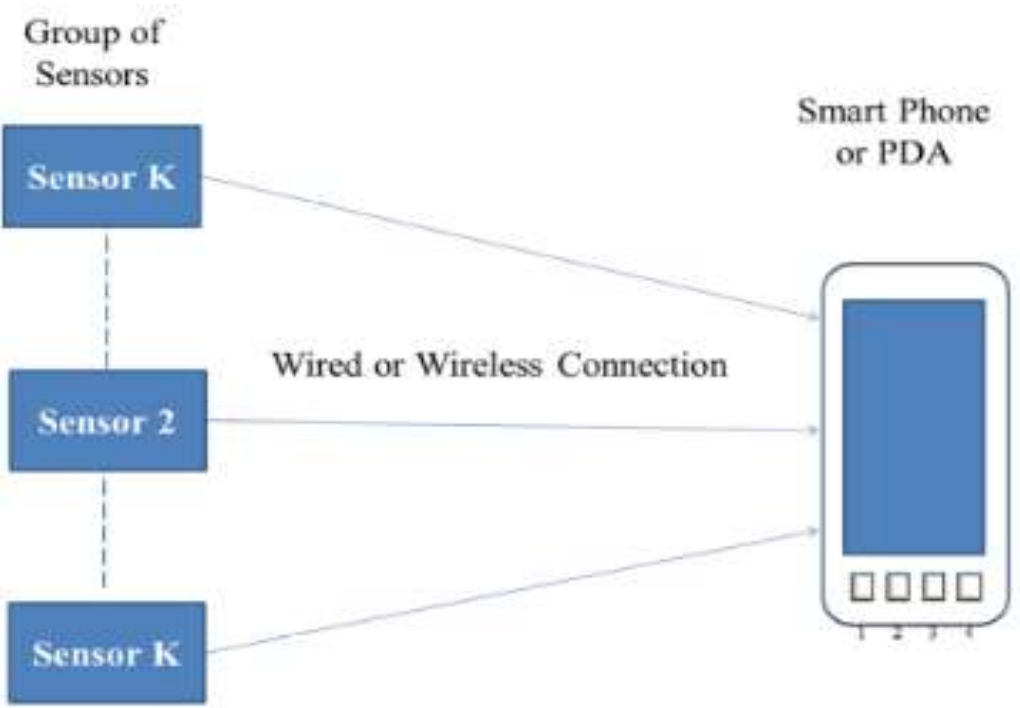

Figure 4. RHMS Architecture- "No Network Access System"[42]

In the second group, i.e. "Direct Network Access System" RHMS architecture, the network accessibility is direct without any intermediate device, as shown in figure 5. The network interface is on-board within the device. Sensors through wired / wireless medium ports data to the coordinator, which in turn uploads the data directly to the remote server. The data analytics and other relevant tasks are accomplished at a remote station. These systems follow the second category of the RHMS architecture and are a relatively open system, i.e. that data is available beyond the patient device. In [48, 50], authors have proposed RHMS following this architecture. In [48], a system is proposed to automate the data coming from sensor nodes and transfer the data to the cloud. This automation includes aggregation, processing and distribution of patient medical data at the backend. The data from heterogeneous sensors are available for doctors and caregivers through predefined exchange services. The system discussed in [49] uses a remote monitoring device to collect the data from the human body and transfer data to the health care center with the help of any of the available wireless interface. An optimal schedule is devised to maintain a queue for buffering of patient data and select a network among available networks; the system also provisions patient mobility. In [50], the system is primarily for diabetic patients and collects the data coming from sensors and transfers it to the webserver. Data-driven and domain expert logic is executed at the remote server.

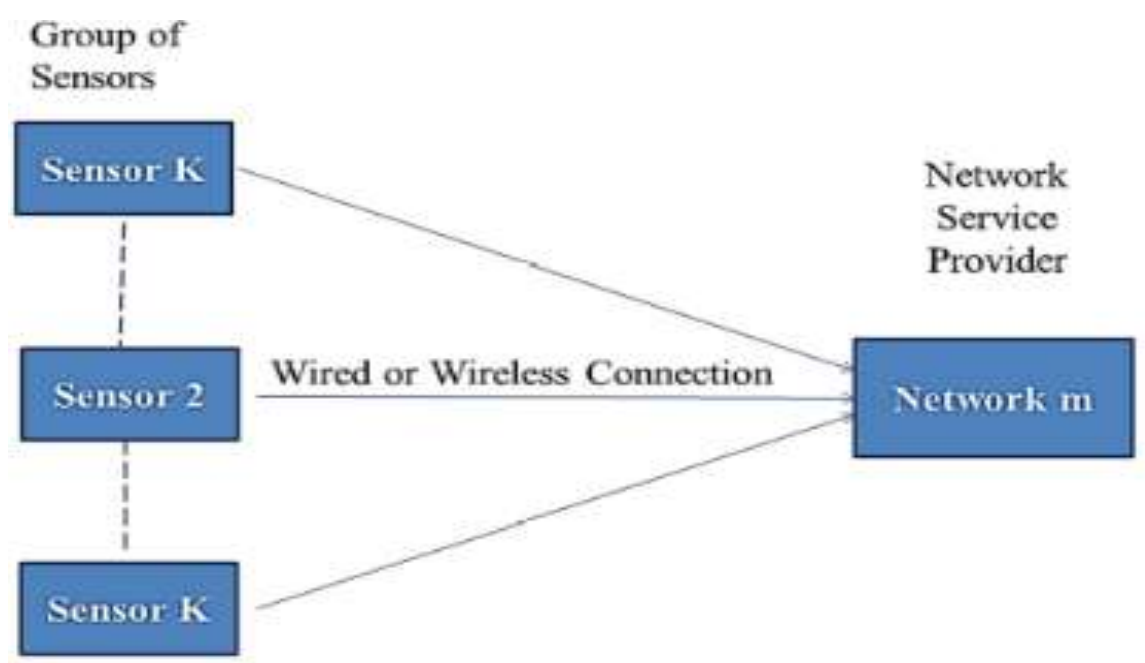

Figure 5. RHMS Architecture- "Direct Network Access System"[42]

The third group refers to "Network Access through Gateway" RHMS Architecture, where there is a use of a dedicated intermediate device as a gateway, smart phone or PDA or simple cellular phone as shown in fig. 6. The gateway stores and transmits the data to the remote server using standard dedicated API and services. This gateway device has numerous facilities such as data storing, filtering, data forwarding to server et cetera. The data actuators, in some instances, also form the multi-hop ad-hoc routing network alongside the gateway for data transmission. 


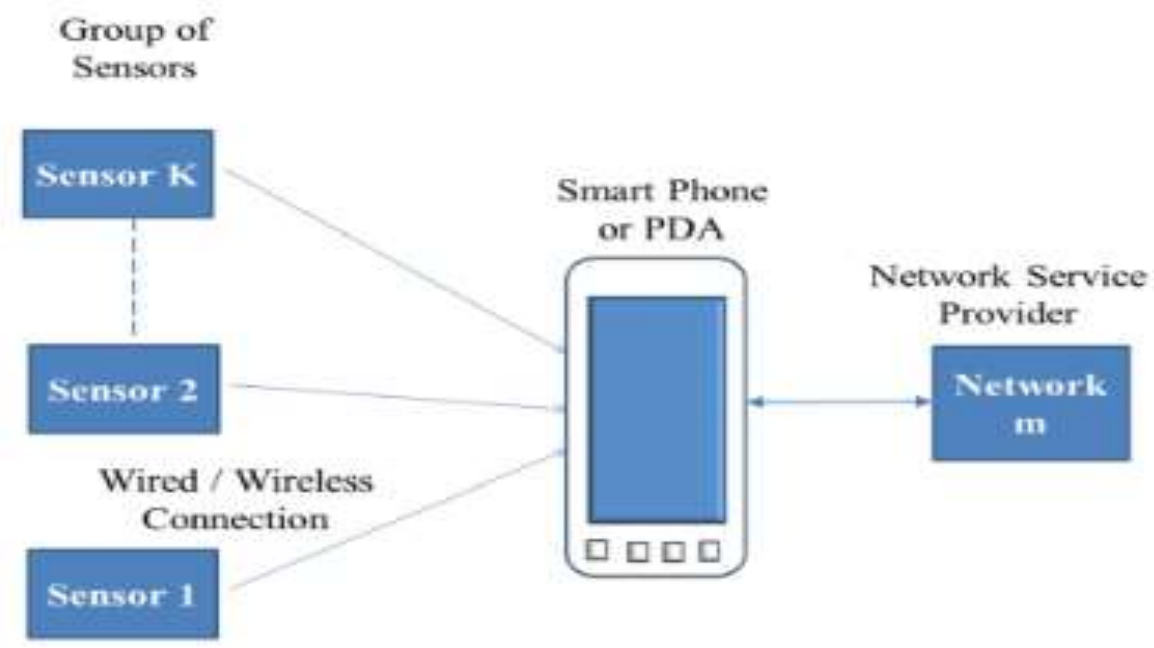

Figure 6. RHMS Architecture- "Network Access through Gateway"[42]

\subsection{ENABLING TECHNOLOGIES}

A standard IoT system consists of sensors, communication interfaces, advanced algorithms, and cloud interface. Sensors are used to get data from various devices. RFID technology and WSN technologies provide the means of communications and network [29]. From this context, in RHMS, usually, patient's medical data are captured by using sensors and transmitted to the server via different networks including BAN, LAN, WAN wired or wireless network. These networks are responsible for accessing and establishing the connections between several system subunits to facilitate an end to end communication from the client end to the server end. Accessing and connecting to remote server/services in the remote healthcare system may be either directly from the client end through a predefined link or a gateway. There are several enabling technologies concerning IoT-based healthcare solutions, and hence it is challenging to provide an explicit list [11]. In this concern, the discussion concentrates only on selected technologies of our interest that suit our specific use case. These enabling technologies are wearable devices, communication networks, big data and cloud computing. Other enabling technologies such as augmented reality, ambient intelligence et cetera are beyond the scope of our use case.

\subsection{Wearable Devices and Communication Networks}

Anything which is uniquely identifiable, able to communicate (can be discovered, receive and reply to messages) and that can perform necessary computations can be used for IoT technology. One of the most adaptive technologies in HCS is the use of sensors in collecting useful data for monitoring and evaluation purpose [51]. Because sensors are more economical, easy to install and cheaper than ever, which becomes an ideal opportunity to make any object smarter. They are available in smart wearable devices like Google smart watch, wristbands and many more others. These wearable devices can be used to measure vital parameters from the outpatient's body and send to the appropriate receiver[52, 53]. This reason makes sensors quickly adopted as the IoT enabling technology in the delivery of healthcare services.

Recent progress in implanted and wearable health monitoring technologies have a powerful potential to change the future of healthcare services through facilitating ubiquitous patients' monitoring. A standard health monitoring system comprises a network of implanted or wearable sensors that continuously monitor physiological parameters. Obtained data are transmitted using existing wireless communication protocols to a base station for further processing [54].

All the technologies that support the interaction of the smart objects are part of the physical infrastructure of the IoT-based healthcare network. Usually, is a combination of various networks ranging from networks for short-range communications (e.g., WPANs, WBANs, WLANs, 6LoWPANs, and WSNs) to long-range communications (e.g., any cellular network such as GSM). Besides, the employment of ultra-wideband (UWB), BLE, NFC, and RFID technologies can help design low-power medical sensor devices as well as communications protocols [11] The notable enhancement contributed by wireless technology is the minimization of the complexity to harness wired transmission through the installation of controllers, sensors, and actuators [55].

\subsection{Cloud Computing and Big Data}

The combination of cloud computing in IoT-based healthcare technologies provides facilities with ubiquitous access to distributed resources, administering services upon on-demand across the network and performing operations to satisfy various requirements. 
Big data can contain vast amounts of vital health data created from numerous medical sensors and present tools for enhancing the effectiveness of allied health diagnosis and monitoring methods [56].

A stand-alone solution is questionable to be satisfactory in addressing the problem of the practitioner shortage. The preparation of fresh doctors takes up to a decade. Consequently, necessary, that various means to solve this deficit in supply are begun from today and progressing forward. In this context, systematically, taking care of patients' health through health monitoring systems is possible, which can be done using modern information and communication technologies (ICT). Therefore, Proactively, offloading some tasks to patients in the monitoring process, which can be a matter of great advantage.

In both developing and developed countries, mobile phones are commonly playing a vital role in a wide range of public health initiatives. In 2011, an action was taken to encourage the adoption of mHealth for tobacco control in developing countries [11]. However, most mHealth projects in developing countries have used text messages service namely Short Message Service (SMS) mainly for increased awareness and communication campaigns and focused mainly on HIV, malaria, and MCH[57], [59]. It is recommendable that all target countries integrate the adoption of ICT into their national health information systems and infrastructure by 2015 [60].

\subsection{PROPOSED SIMPLIFIED MODEL FOR RHMS}

Proposed RHMS emphasizes on simplicity while trying to achieve the core functionality of an ideal RHMS. As shown in figure 8, it is a hybrid of two RHMS architecture, namely DNAS and NATG, with design to offer flexibility and redundancy capabilities. To meet the standard requirement of the RHMS, the proposed RHMS comprises of three layers, namely data acquisition, data transmission and data processing.

A wearable device performs Data Acquisition via in-built multiple sensors that measure physiological biomarkers, such as ECG, skin temperature, respiratory rate, EMG muscle activity, and gait (posture). In this case, we consider affordable wearable devices such as smart watches or wristbands with the supporting capability of BLE technology. The sensors connect to the network through an intermediate data aggregator or concentrator, for our case is a BLE enabled mobile phone located in the vicinity of the outpatient that supports SMS functionality within GSM network infrastructure.

For Data Transmission, the BLE and GSM technologies are the components of the system that are responsible for conveying patient's recordings from any remote location to the Healthcare's data centre with the guaranteed privacy and security, ideally in near real-time. GSM network in our study facilitates the sending information process using SMS from the mobile phone or a wearable device with SIM support capability to the doctor's server via Base Transceiver Station (BTS). We adopt SMS in this study due to the cost and ubiquitous aspects of SMS. SMS is a top-rated wireless service offered by GSM network which comprises the most significant worldwide number of users throughout the world. It is the transmission of an alphanumeric message between two parties [61]. In mobile communication, the SMS is one of the superior and well-tried services with global availability in the GSM networks. 


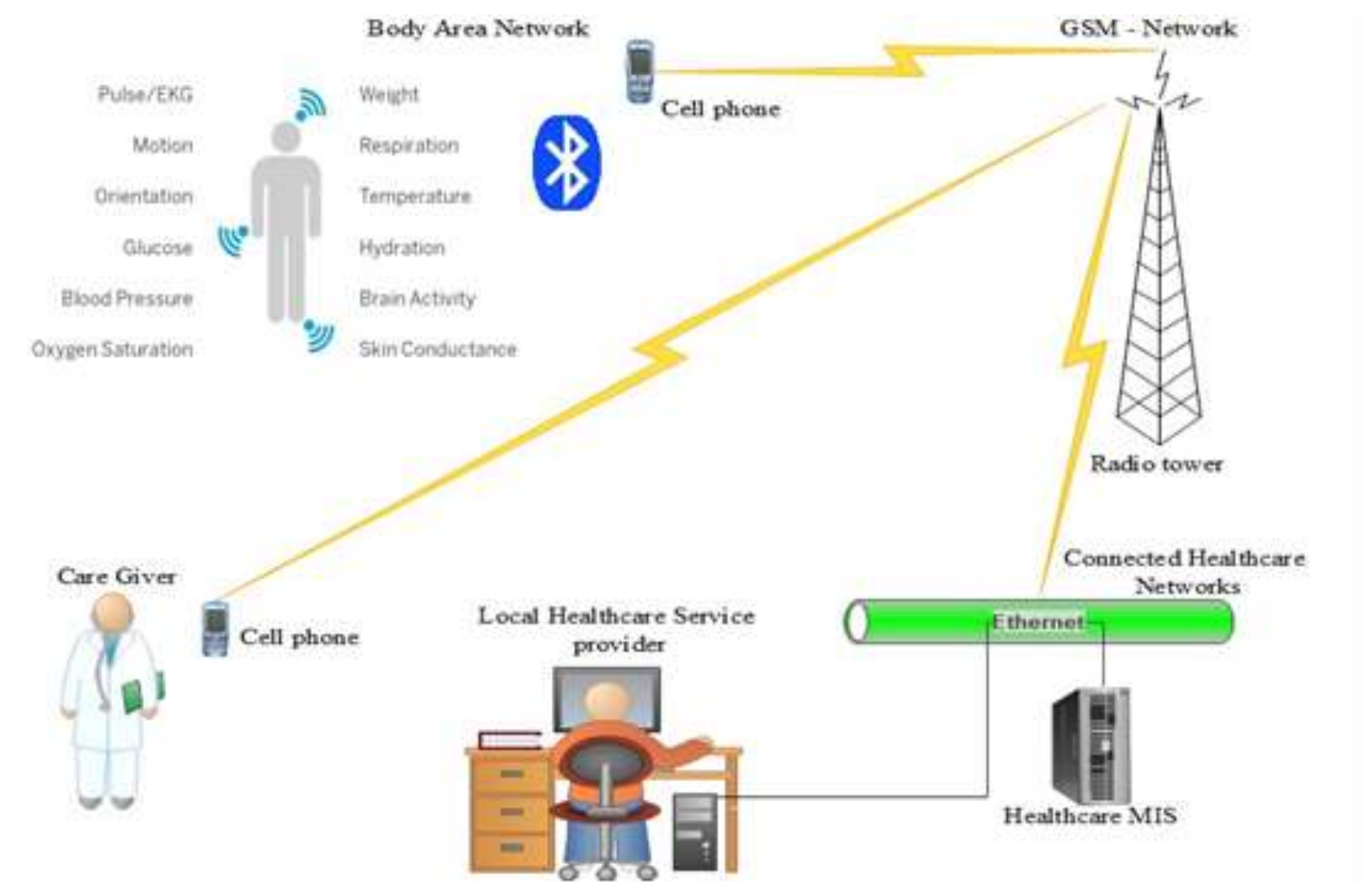

Figure 8. A simplified RHMS

In Data Processing, the system relies on the healthcare center resources for its functionality, which can also allow an extension to the region or country level. It comprises of three components to meet the standard requirements of cloud processing as in advanced RHMS: storage, analytics, and visualization. It is done by doctors/medical assistants at the local healthcare center using the available resources by processing and give the results to the users in a real-time. Fortunately, the existing local health care centers have interconnection with at least a referral hospital, ministry of health or any respective health stakeholders within a particular region or country at large. This interconnection is to comply with current health policy for improving healthcare services deliverance through information exchanging in several issues regarding referral cases, diseases out breaking, insurance et cetera. Our system leverages this context by offloading some tasks which are beyond the capacity of the respective local healthcare center to region referral hospitals or national health research centers within a country. For instance, data analytics and visualization, which need complex and expensive systems can be offloaded from local healthcare centers while the local health care center can manage other tasks such as data storage.

As a result, our designed system even with constrained ICT and healthcare resources by efficiently using and taking advantage of the existing infrastructure and resources can suit for long term storage of patient's biomedical information as well assisting health professionals with diagnostic information. The system can advise and alerts in real-time the doctors/medical assistants about the changing of vital parameters in order to take preventive measures.

By incorporating the use of ICT, especially IoT applications in Healthcare systems can bring relief to the soaring healthcare services deliverance. For instance, in more critical scenarios such as rural settings where remote health monitoring is vital for general life safety and well-being of people. Adopting a simplified model, as shown in figure 7 by leveraging the use of existing technology and infrastructure can be a significant step as a transition strategy towards a complete IoT model deployment in delivering healthcare services. The emphasis of this paper bases on this aspect by leveraging ubiquitous computing in enabling remote health monitoring to improve healthcare services deliverance in local remote areas with limited ICT infrastructure and healthcare services. 


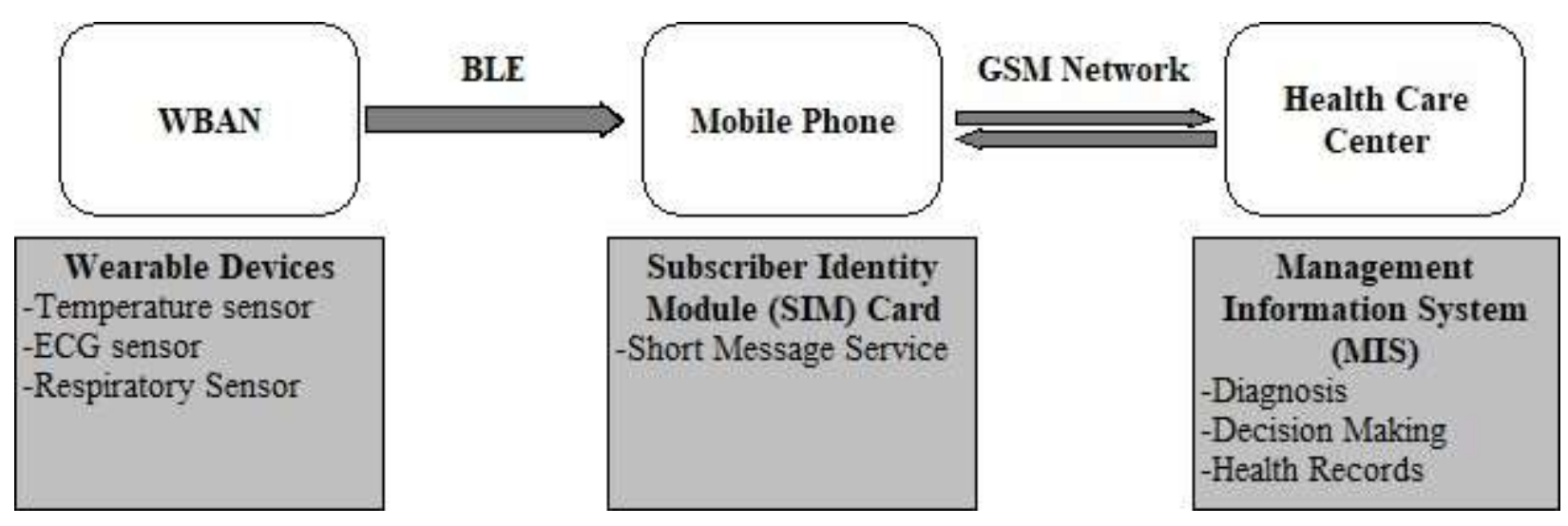

Figure 7. A simplified model for remote health monitoring system

\subsection{RHSM IMPLEMENTATION CONCEPT AND EVALUATION}

\subsection{Data acquisition from BAN (Sensor devices), Base Station [BS) and Mobile Station (MS)}

Signals in this case are multipath in nature. The channel comprise none obstructed line of sight(LOS) and an obstructed line of sight (NLOS).Each path produces a replica of the signal of each other which is attenuated by free space or scatters and its delay.

From the theory of linear system, the attenuation is the scaling factor and the delay corresponds to a function $\delta\left(t-\tau_{0}\right)$ [22] Therefore $a_{L-1} \delta\left(t-\tau_{L-1}\right)$ is the impulse response where $a_{L-1}$ is the $1^{\text {th }}$ path or link and $\delta\left(t-\tau_{L-1}\right)$ is the attenuation factor and delay respectively.

The impulse response of the wireless communication channel is simply the combination of all these paths which link the BAN devices and the cell phone devices to the BS given as $h(t)$ is given as $h(t)=a_{0} \delta\left(t-\tau_{0}\right)+a_{1} \delta\left(t-\tau_{1}\right)+\ldots \ldots \ldots .+a_{L-1} \delta\left(t-\tau_{L-1}\right)$

$$
\sum_{i=0}^{L-1} a_{i} \delta\left(t-\tau_{i}\right)
$$

$a_{0} \delta\left(t-\tau_{0}\right)$ is a multipath component which is really a wireless channel carrying carrying data from the patient through one of the devices of BAN (Sensor network). Index 0 is a LOS while others are NLOS.

The wireless signal transmitted by the Base station (BS) to doctor's Mobile station is simply given as $s(t)=\mathfrak{R} e\left\{S_{b}(t) e^{j 2 \pi f_{c} t}\right\}$ which is the Baseband/Pasband representation. Therefore the expected net received signal is $y(t)=h(t) * s(t)$ where $h(t)$ is an impulse response and $s(t)$ is the transmitted signal. This can simply be rewritten as

$$
y(t)=\mathfrak{R} e\left\{\left\{\sum_{i=0}^{L-1} a_{i} S_{b}\left(t-\tau_{i}\right) e^{-j 2 \pi f_{c} \tau_{i}}\right\} e^{j 2 \pi f_{c} t}\right\}
$$

The received power between cell phone $m$ and wearable device $S$, in the BAN is given by

$$
P_{r m}=P_{t s} \frac{L}{d^{\eta}}
$$


Where $P_{r m}$ denotes received power of cell phone device and $P_{t s}$ denotes transmitted power of wearable device. $L$ represents the path-loss constant factor and $d$ represents the distance between sensor devices in the BAN and cell phone device. Parameter $\eta$ represents the path-loss exponent.

The link transmission rate between $s$ and $m$ is given as $R_{s, m}=B \log _{2}(1+k)$ where $B$ denotes the transmission bandwidth and $k$ is the signal to noise ratio $S N R$ obtained as $k=\frac{L P_{t s}}{N_{o} B_{s, m} d_{s, m}^{\eta}}$. This can further expressed as

$$
R_{s, m}=B_{s, m} \log _{2}\left(1+\frac{L P_{t s}}{N_{o} B_{s, m} d_{s, m}^{\eta}}\right)
$$

Where $B_{s, m}$ is the bandwidth between wearable device and cell phone device, $P_{t s}$ is the transmission power of cell phone devices, $d_{s, m}$ is the distance between m sensor devices from the BAN and the cell phone device. $L$ and $\eta$ are considered the same for all the communication link for simplicity although in reality $L$ parameter may be different from device to device in a cellular communication. $N_{o}$ is the additive white Gaussian noise (AWGN).

The transmission time from the sensor device $s$ to cell phone device $m$ is given as

$$
T_{s, m}^{t r}=\frac{\lambda_{s}}{R_{s, m}}
$$

where $\lambda_{s}$ denotes data size of device $s$ and $R_{s, m}$ is the transmission rate between device $s$ and $m$.

\subsection{CONCLUSION AND FUTURE WORK}

This paper presents a simplified system model for remote monitoring of the outpatient's well-being in the limited Internet of Things (IoT) infrastructure. Adopting a simplified model can be a significant step as a transition strategy towards a complete IoT model for RHMS in delivering healthcare services. This paper emphasis on this aspect by leveraging ubiquitous computing in enabling remote health monitoring to improve healthcare services deliverance in local remote areas with limited ICT infrastructure and healthcare services. Through a survey of several RHMS and their architecture, the paper propose a flexible, simplified RHMS for constrained IoT infrastructure by leveraging the existing resources and IT infrastructure to achieve the primary goal of RHMS. The design is cheap and easily deployable for health remote monitoring purposes as it can monitor outpatient's well-being in realtime. The system advises and alerts in real-time the doctors/medical assistants about the changing of vital parameters in order to take preventive measures. For future work, the model can be extended to incorporate other functionalities such as tracking symptoms, emotions, and sleep patterns. Also integration of security and privacy capabilitieswhich is out of the scope in our design can be a meaningful future work. Security and privacy of patients and managerial data is still a challenge to most of the healthcare industries. This is essential for full adoption of IoT in healthcare applications. Hence, there is a need to incorporate these aspects in designing and implementation of IoT based healthcare systems, regardless of a scenario with limited IT infrastructure that can smoothly support IoT use cases implementation.

\section{REFERENCES}

[1] T. Aamc, "Myths and Facts : The Physician Shortage," 1997.

[2] R. M. Scheffler, J. X. Liu, and M. R. Dal, "Forecasting the global shortage of physicians : an economic- and needs-based approach," 2015.

[3] "A framework for a systems approach to health care delivery," in Building a Better Delivery System: A New Engineering/Health Care Partnership, 2005.

[4] Anon, "Health care spending per privately insured American increased 7.4 percent in 2003," News-Medical.Net. 2004.

[5] G. F. Sheldon, T. C. Ricketts, A. Charles, J. King, E. P. Fraher, and A. Meyer, "The Global Health Workforce Shortage : Role of Surgeons and Other Providers," vol. 42, pp. 63-85, 2008.

[6] World Health Organization, "World health statistics - Monitoring health for the SDGs," World Heal. Organ., 2016.

[7] G. Todd and M. Mamdani, "Tanzania and the Sustainable Development Goals :," pp. 1-32, 2017.

[8] M. Mamdani and M. Bangser, "Poor people's experiences of health services in Tanzania: a literature review.," Reprod. Health Matters, 2004. 
[9] H. Situation and H. Policies, "United Republic of Tanzania," vol. 34, pp. 17-18, 2017.

[10] J. Qi, P. Yang, G. Min, O. Amft, F. Dong, and L. Xu, “Advanced Internet of Things for Personalised Healthcare System : A Survey," Pervasive Mob. Comput., 2017.

[11] S. M. R. Islam, D. Kwak, and H. Kabir, "The Internet of Things for Health Care : A Comprehensive Survey," IEEE Access, vol. 3, pp. 678-708, 2015.

[12] M. Hassanalieragh, A. Page, T. Soyata, G. Sharma, M. Aktas, and G. Mateos, "Health Monitoring and Management Using Internet-of-Things ( IoT ) Sensing with Cloud-based Processing : Opportunities and Challenges,” 2015.

[13] S. Soundarya, "Smart Health Monitoring System using IoT,” vol. 6, no. 14, pp. 1-2, 2018.

[14] V. M. Rohokale, “A Cooperative Internet of Things ( IoT ) for Rural Healthcare Monitoring and Control,” 2011.

[15] A. Santos, J. Macedo, A. Costa, and M. J. Nicolau, "Internet of Things and Smart Objects for M-Health Monitoring and Control,” Procedia Technol., vol. 16, pp. 1351-1360, 2014.

[16] L. Da Xu, W. He, and S. Li, "Internet of things in industries: A survey," IEEE Transactions on Industrial Informatics. 2014.

[17] A. Lele, "Internet of things (IoT)," in Smart Innovation, Systems and Technologies, 2019.

[18] A. Whitmore, A. Agarwal, and L. Da Xu, "The Internet of Things-A survey of topics and trends," Inf. Syst. Front., 2015.

[19] E. Ahmed et al., "The role of big data analytics in Internet of Things," Comput. Networks, 2017.

[20] Y. YIN, Y. Zeng, X. Chen, and Y. Fan, “The internet of things in healthcare: An overview," Journal of Industrial Information Integration. 2016.

[21] M. Ammar, G. Russello, and B. Crispo, "Internet of Things: A survey on the security of IoT frameworks," J. Inf. Secur. Appl., 2018.

[22] N. Shahid and S. Aneja, "Internet of Things: Vision, application areas and research challenges," in Proceedings of the International Conference on IoT in Social, Mobile, Analytics and Cloud, I-SMAC 2017, 2017.

[23] A. Al-Fuqaha, M. Guizani, M. Mohammadi, M. Aledhari, and M. Ayyash, "Internet of Things: A Survey on Enabling Technologies, Protocols, and Applications," IEEE Commun. Surv. Tutorials, 2015.

[24] GSMA Association, "Understanding the Internet of Things (IoT)," Gsma Connect. Living, 2014.

[25] F. Tao, Y. Cheng, L. Da Xu, L. Zhang, and B. H. Li, "CCIoT-CMfg: Cloud computing and internet of things-based cloud manufacturing service system," IEEE Trans. Ind. Informatics, 2014.

[26] S. D. T. Kelly, N. K. Suryadevara, and S. C. Mukhopadhyay, "Towards the implementation of IoT for environmental condition monitoring in homes," IEEE Sens. J., 2013.

[27] I. Mohanraj, K. Ashokumar, and J. Naren, "Field Monitoring and Automation Using IOT in Agriculture Domain," in Procedia Computer Science, 2016.

[28] M. Swan, "Sensor mania! the internet of things, wearable computing, objective metrics, and the quantified self 2.0," Journal of Sensor and Actuator Networks. 2012.

[29] B. Farahani, F. Firouzi, V. Chang, M. Badaroglu, N. Constant, and K. Mankodiya, "Towards fog-driven IoT eHealth: Promises and challenges of IoT in medicine and healthcare," Futur. Gener. Comput. Syst., 2018.

[30] A. M. Rahmani et al., "Exploiting smart e-Health gateways at the edge of healthcare Internet-of-Things: A fog computing approach," Futur. Gener. Comput. Syst., 2018.

[31] S. Fang et al., "An integrated system for regional environmental monitoring and management based on internet of things," IEEE Trans. Ind. Informatics, 2014.

[32] C. Mathas, "No Title," Digi-Key Electronics. Anline]. https://www.digikey.com/en/articles/techzone/2014/jul/the-role-of-sensors-in-iot-medical-and-healthcare-applications. [Accessed: 11-Nov-2019].

[33] M. Chen, Y. Ma, Y. Li, D. Wu, Y. Zhang, and C. H. Youn, "Wearable 2.0: Enabling Human-Cloud Integration in Next Generation Healthcare Systems," IEEE Commun. Mag., vol. 55, no. 1, pp. 54-61, 2017.

[34] Z. Pang, Q. Chen, J. Tian, L. Zheng, and E. Dubrova, "Ecosystem Analysis in the Design of Open Platform- based InHome Healthcare Terminals towards the," pp. 529-534, 2013.

[35] J. Qi, P. Yang, G. Min, O. Amft, F. Dong, and L. Xu, “Advanced Internet of Things for Personalised Healthcare System : A Survey Advanced internet of things for personalised healthcare systems : A survey," Pervasive Mob. Comput., vol. 41, no. 286545, pp. 132-149, 2017.

[36] M. Talal et al., "Smart Home-based IoT for Real-time and Secure Remote Health Monitoring of Triage and Priority System using Body Sensors: Multi-driven Systematic Review,” Journal of Medical Systems. 2019.

[37] P. R. Newswire, "The mHealth (Mobile Healthcare) Ecosystem: 2018 - 2030 - Opportunities, Challenges, Strategies \& Forecasts," LON-REPORTBUYER. 2018.

[38] P. R. Newswire, "The mHealth (Mobile Healthcare) Ecosystem: 2015 - 2030 - Opportunities, Challenges, Strategies \& Forecasts - Reportlinker Review," NY-Reportlinker. 2015.

[39] S. T. Ben Hamida, E. Ben Hamida, and B. Ahmed, "A new mHealth communication framework for use in wearable 
WBANs and mobile technologies," Sensors (Switzerland), 2015.

[40] E. Kartsakli et al., "A survey on M2M systems for mhealth: a wireless communications perspective," Sensors (Switzerland). 2014.

[41] A. P. Abidoye, N. A. Azeez, A. O. Adesina, K. K. Agbele, and H. O. Nyongesa, "Using Wearable Sensors for Remote Healthcare Monitoring System,” J. Sens. Technol., 2011.

[42] R. Mishra and R. Pandey, "Aspects of Network Architecture for Remote Healthcare Systems."

[43] P. Pawar, V. Jones, B. J. F. van Beijnum, and H. Hermens, "A framework for the comparison of mobile patient monitoring systems," J. Biomed. Inform., 2012.

[44] M. Aminian, “A Hospital Healthcare Monitoring System Using Wireless Sensor Networks," J. Heal. Med. Informatics, 2013.

[45] J. H. Kim, R. Roberge, J. B. Powell, A. B. Shafer, and W. Jon Williams, "Measurement accuracy of heart rate and respiratory rate during graded exercise and sustained exercise in the heat using the zephyr BioHarness TM," Int. J. Sports Med., 2013.

[46] P. S. Pandian et al., "Smart Vest: Wearable multi-parameter remote physiological monitoring system," Med. Eng. Phys., 2008.

[47] N. Oliver and F. Flores-Mangas, "HealthGear: A real-time wearable system for monitoring and analyzing physiological signals," in Proceedings - BSN 2006: International Workshop on Wearable and Implantable Body Sensor Networks, 2006.

[48] S. H. Shah, A. Iqbal, and S. S. A. Shah, "Remote health monitoring through an integration of wireless sensor networks, mobile phones \& Cloud Computing technologies," in Proceedings of the 3rd IEEE Global Humanitarian Technology Conference, GHTC 2013, 2013.

[49] D. Niyato, E. Hossain, and S. Camorlinga, "Remote patient monitoring service using heterogeneous wireless access networks: Architecture and optimization," IEEE J. Sel. Areas Commun., 2009.

[50] M. K. Suh et al., "Dynamic self-adaptive remote health monitoring system for diabetics," in Proceedings of the Annual International Conference of the IEEE Engineering in Medicine and Biology Society, EMBS, 2012.

[51] N. C. Luong, D. T. Hoang, P. Wang, D. Niyato, D. I. Kim, and Z. Han, "Data Collection and Wireless Communication in Internet of Things (IoT) Using Economic Analysis and Pricing Models: A Survey," IEEE Communications Surveys and Tutorials. 2016.

[52] J. Ko, C. Lu, M. B. Srivastava, J. A. Stankovic, A. Terzis, and M. Welsh, "Wireless sensor networks for healthcare," in Proceedings of the IEEE, 2010.

[53] A. E. Boualouache, O. Nouali, S. Moussaoui, and A. Derder, "A BLE-based data collection system for IoT," in NTIC 2015 - 2015 1st International Conference on New Technologies of Information and Communication, Proceeding, 2015.

[54] M. Ghamari, B. Janko, R. S. Sherratt, W. Harwin, R. Piechockic, and C. Soltanpur, "A survey on wireless body area networks for ehealthcare systems in residential environments," Sensors (Switzerland), 2016.

[55] H. Ghayvat, S. Mukhopadhyay, X. Gui, and N. Suryadevara, "WSN- and IOT-based smart homes and their extension to smart buildings," Sensors (Switzerland), 2015.

[56] S. M. R. Islam, D. Kwak, M. H. Kabir, M. Hossain, and K. S. Kwak, "The internet of things for health care: A comprehensive survey," IEEE Access, vol. 3, pp. 678-708, 2015.

[57] M. S. C. Lim, J. S. Hocking, M. E. Hellard, and C. K. Aitken, "SMS STI: A review of the uses of mobile phone text messaging in sexual health," International Journal of STD and AIDS. 2008.

[58] C. M. Coomes, M. A. Lewis, J. D. Uhrig, R. D. Furberg, J. L. Harris, and C. M. Bann, "Beyond reminders: a conceptual framework for using short message service to promote prevention and improve healthcare quality and clinical outcomes for people living with HIV.," AIDS care. 2012.

[59] C. Déglise, L. Suzanne Suggs, and P. Odermatt, "SMS for disease control in developing countries: A systematic review of mobile health applications," Journal of Telemedicine and Telecare. 2012.

[60] T. ( U. of A. Ahlin and M. ( U. of A. Nichter, "Takes a Stand: e/m-Health and Telemedicine." [Online]. Available: http://cagh.medanthro.net/take-a-stand-policy-statements-by-cagh/takes-a-stand-em-health-and-telemedicine/. [Accessed: 11-Nov-2019].

[61] C. L. Tseng et al., "Feasibility study on application of GSM-SMS technology to field data acquisition," Comput. Electron. Agric., 2006. 